\title{
Tunneling-percolation origin of nonuniversality: Theory and experiments
}

\author{
Sonia Vionnet-Menot, ${ }^{1}$ Claudio Grimaldi, ${ }^{1, *}$ Thomas Maeder, ${ }^{1,2}$ Sigfrid Strässler, ${ }^{1,2}$ and Peter Ryser ${ }^{1}$ \\ ${ }^{1}$ Laboratoire de Production Microtechnique, Ecole Polytechnique Fédérale de Lausanne, Station 17, CH-1015 Lausanne, Switzerland \\ ${ }^{2}$ Sensile Technologies SA, PSE, CH-1015 Lausanne, Switzerland \\ (Received 25 July 2004; revised manuscript received 10 November 2004; published 16 February 2005)
}

\begin{abstract}
A vast class of disordered conducting-insulating compounds close to the percolation threshold is characterized by nonuniversal values of transport critical exponent $t$, in disagreement with the standard theory of percolation which predicts $t \simeq 2.0$ for all three-dimensional systems. Various models have been proposed in order to explain the origin of such universality breakdown. Among them, the tunneling-percolation model calls into play tunneling processes between conducting particles which, under some general circumstances, could lead to transport exponents dependent of the mean tunneling distance $a$. The validity of such theory could be tested by changing the parameter $a$ by means of an applied mechanical strain. We have applied this idea to universal and nonuniversal $\mathrm{RuO}_{2}$-glass composites. We show that when $t>2$ the measured piezoresistive response $\Gamma$, i.e., the relative change of resistivity under applied strain, diverges logarithmically at the percolation threshold, while for $t \simeq 2, \Gamma$ does not show an appreciable dependence upon the $\mathrm{RuO}_{2}$ volume fraction. These results are consistent with a mean tunneling dependence of the nonuniversal transport exponent as predicted by the tunneling-percolation model. The experimental results are compared with analytical and numerical calculations on a random-resistor network model of tunneling percolation.
\end{abstract}

DOI: 10.1103/PhysRevB.71.064201

\section{INTRODUCTION}

Despite the fact that transport properties of disordered insulator-conductor composites have been studied for more than thirty years, some phenomena still remain incompletely understood. One such phenomenon is certainly the origin of nonuniversality of the dc transport near the conductorinsulator critical transition. According to the standard theory of transport in isotropic percolating materials, the bulk conductivity $\sigma$ of a composite with volume concentration $x$ of the conducting phase behaves as a power law of the form ${ }^{1,2}$

$$
\sigma \simeq \sigma_{0}\left(x-x_{c}\right)^{t},
$$

where $\sigma_{0}$ is a proportionality constant, $x_{c}$ is the critical concentration below which the composite has zero conductivity (or more precisely the conductivity of the insulating phase), and $t$ is the $\mathrm{dc}$ transport critical exponent. The above expression holds true in the critical region $x-x_{c} \ll 1$ in which critical fluctuations extend over distances much larger than the characteristic size of the constituents. As a consequence, contrary to $\sigma_{0}$ and $x_{c}$ which depend on microscopic details such as the microstructure and the mean intergrain junction conductance, the exponent $t$ is expected to be material independent. ${ }^{1,2}$ The universality of $t$ is indeed confirmed by various numerical calculations of random-resistor network models which have established that $t=t_{0} \simeq 2.0$ for threedimensional lattices to a rather high accuracy. ${ }^{3-5}$

Confirmations to the standard percolation theory of transport universality are found only in a limited number of experiments on real disordered composites. This is illustrated in Fig. 1, where we have collected 99 different values of the critical exponent $t$ and the critical threshold $x_{c}$ measured in various composites including carbon-black-polymer systems, ${ }^{6-26}$ oxide-based thick film resistors (TFRs), ${ }^{27-38}$ and other metal-inorganic and -organic insulator composites. ${ }^{26,39-48}$ It is clear that, despite that many of the
PACS number(s): 72.20.Fr, 64.60.Fr, 72.60. $+\mathrm{g}$

$t$-values reported in Fig. 1 are close to $t_{0} \simeq 2.0$, almost $50 \%$ of the measured critical exponents deviate from universality by displaying $t \neq t_{0}$.

Examining all the data reported in Fig. 1, one observes that the lack of universality is not limited to a particular class of materials, although the granular metals (empty diamonds in Fig. 1) have somewhat less spread values of $t$ compared to the carbon-black and TFRs composites. Another important

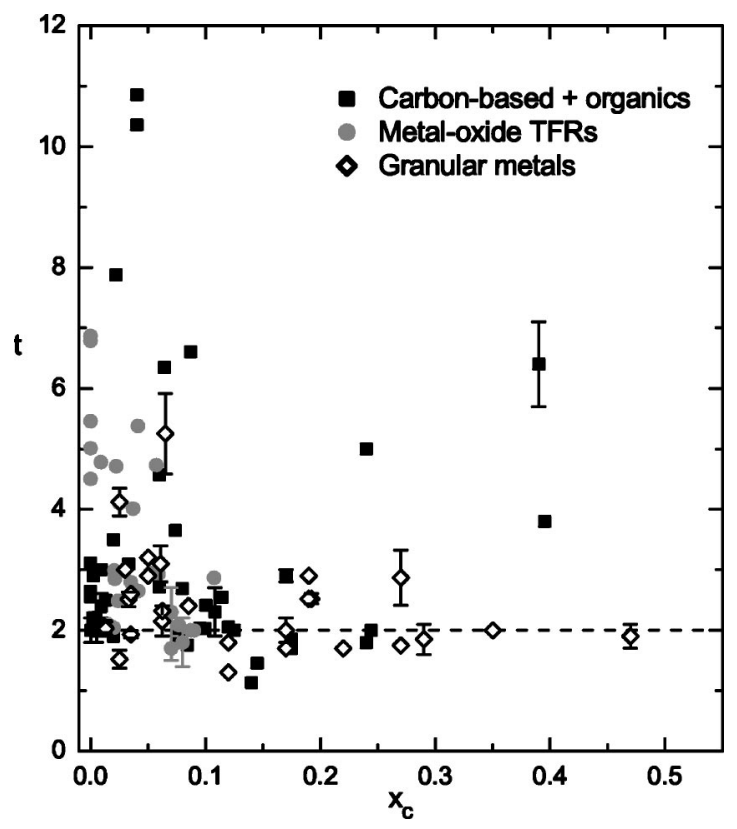

FIG. 1. Collection of critical exponent values $t$ and corresponding critical threshold concentration $x_{c}$ for various disordered conductor-insulator composites. Carbon-black-polymer systems are from Refs. 6-26, oxide-based thick film resistors are from Refs. 27-38, metal-inorganic and -organic insulator granular metals are from Refs. 26 and 39-48. The dashed line denotes the universal value $t_{0} \simeq 2.0$. 
observation is that for the vast majority of the cases, the nonuniversal critical exponent is larger than $t_{0}$, and only few data display $t<t_{0}$. Finally, there is no clear correlation between $t$ and the critical concentration value $x_{c}$.

With the accumulation of experimental reports of nonuniversality, various theories have been proposed in order to find an origin to this phenomenon. ${ }^{9,49-52}$ In Ref. 9 it was argued that, in carbon-black-polymer composites, long-range interactions could drive the system towards the mean-field regime for which $t=t_{\mathrm{MF}}=3.0$. This interpretation cannot, however, explain the observation of critical exponents much larger than $t_{\mathrm{MF}}$, such as those of carbon-based composites or TFRs which display values of $t$ as high as $t \simeq 5-10$. The authors of Ref. 50 introduced the random-void (RV) model of continuum percolation where current flows through a conducting medium embedding insulating spheres placed at random. By using an earlier result, ${ }^{49}$ they were able to show that for this model dc transport is described by a universality class different from that of standard percolation model on a lattice. The resulting critical exponent was found to be $t$ $\simeq 2.4$ for three-dimensional systems. More recently, Balberg generalized the RV model in an attempt to explain higher $t$ values. ${ }^{52}$ The same author also proposed a model of transport nonuniversality based on an inverted RV model in which current flows through tunneling processes between conducting spheres immersed in an insulating medium. ${ }^{51}$ Within this picture, if the distribution function of the tunneling distances decays much slower than the tunneling decay, then the critical exponent becomes dependent on the mean tunneling distance $a$ and, in principle, has no upper bound.

In addition to the above models, there were also explications pointing out that when Eq. (1) is used to fit experimental data not restricted to the critical region, "apparent critical exponents," usually larger than $t_{0}$, could be misinterpreted as real critical exponents. ${ }^{37,53}$ Although this possibility cannot be excluded for some of the data reported in Fig. 1, it is however, quite unrealistic to identify the whole set of reported nonuniversal exponents as merely apparent.

The mean-field interpretation, ${ }^{9}$ the RV model and its generalization, ${ }^{50,52}$ and the tunneling inverted RV model (also known as the tunneling-percolation model), ${ }^{51}$ have been devised to describe nonuniversality for various classes of materials. For example, the RV model applies in principle to composites where the linear size of the conducting particles is much smaller than that of the insulating grains so that the conducting phase can be approximated by a continuum. The tunneling-percolation model has been instead conceived to apply to those composites for which intergrain tunneling is the main microscopic mechanism of transport, such as in carbon-black-polymer composites,${ }^{54}$ or in oxidebased TRFs. ${ }^{55-57}$ In principle, the two models could even coexist together if the continuum phase of a RV system is made of nonsintered conducting particles interacting through tunneling processes.

In this situation, the different proposed theories could account for the variety of nonuniversal exponents shown in Fig. 1. However, it is also true that, in order to identify a given mechanism of nonuniversality for specific composites, little has been done beyond a mere fit to Eq. (1). For example, in Ref. 52 the dc critical exponents have been exam- ined together with the relative resistance noise exponent, and in Ref. 39 a study on ac and magnetoresistive exponents for various composites has been presented. As a matter of fact, no conclusive answers have been reached and the different models of nonuniversality listed above have not proven to really apply to real composites.

In this paper we present our contribution to the understanding of the origin of transport nonuniversality by attacking the problem from a different point of view. In contrast with the mean-field hypothesis, ${ }^{9}$ the RV model, ${ }^{50}$ and its extension, ${ }^{52}$ the tunneling-percolation model of Balberg predicts that the critical exponent $t$ acquires an explicit dependence upon a microscopic variable (the mean-tunneling distance a) which could be altered by a suitable external perturbation. So, if transport nonuniversality is driven by tunneling, it would be possible to change the value of the transport critical exponent $t$ by applying a pressure or a strain to the composite. Conversely, when a material belongs to some universality class (standard percolation theory, meanfield universality class, or the RV model) its exponent is expected to be independent of microscopic details and an applied strain would not change $t$.

We have applied this idea to $\mathrm{RuO}_{2}$-based TFRs whose transport properties are known to be governed by intergrain tunneling processes. ${ }^{55-57}$ In the following of this paper we show that the behavior of the piezoresistive response, i.e., the change of resistivity upon applied mechanical strain, ${ }^{54}$ as a function of concentration $x$ of $\mathrm{RuO}_{2}$, can be interpreted as due to a tunneling distance dependence of the dc critical exponent $t$, as originally proposed in Ref. 51 . The paper is organized as follows. In the next section we briefly review the tunneling-percolation theory and the RV model and its extension. In Sec. III we describe the theory of piezoresistivity for percolating composites and in Sec. IV we present our experimental results. The last section is devoted to a discussion and to the conclusions.

\section{MODELS OF NONUNIVERSALITY}

The RV models, ${ }^{50,52}$ and the tunneling-percolation theory, ${ }^{51}$ have one point in common. They all rely on the work of Kogut and Straley who first developed a theoretical model of nonuniversality based on random-resistor networks. ${ }^{49}$ In this model, to each neighboring couple of sites on a regular lattice it is assigned with probability $p$ a bond with conductance $g \neq 0$ and bond with $g=0$ with probability $1-p$. The resulting bond conductance distribution function is then

$$
\rho(g)=p h(g)+(1-p) \delta(g),
$$

where $\delta(g)$ is the Dirac delta function and $h(g)$ is the distribution function of the finite bond conductances. Close to the bond percolation threshold $p_{c}$, the conductivity $\Sigma$ of the network behaves as

$$
\Sigma=\Sigma_{0}\left(p-p_{c}\right)^{t},
$$

where $\Sigma_{0}$ is a prefactor. In this and in the subsequent section we distinguish the conductivity $\Sigma$ of a random-resistor network from that of real composites [Eq. (1)]. When $h(g)$ 
$=\delta\left(g-g_{0}\right)$ where $g_{0}$ is some nonzero value, Eq. (2) reduces to the standard bimodal model for which, close to the percolation threshold, the network conductivity $\Sigma$ follows Eq. (3) with $t=t_{0} \simeq 2.0$ for all three-dimensional lattices. Instead, if $h(g)$ has a power law divergence for small $g$ of the form

$$
\lim _{g \rightarrow 0} h(g) \propto g^{-\alpha},
$$

where $\alpha \leqslant 1$, then universality is lost for sufficiently large values of the exponent $\alpha .{ }^{49}$ For lattices of dimension $D$, the resulting conductivity critical exponent is ${ }^{58-60}$

$$
t= \begin{cases}t_{0} & \text { if }(D-2) \nu+\frac{1}{1-\alpha}<t_{0}, \\ (D-2) \nu+\frac{1}{1-\alpha} & \text { if }(D-2) \nu+\frac{1}{1-\alpha}>t_{0},\end{cases}
$$

where $t_{0}$ is the universal value and $\nu$ is the correlation-length exponent $(\nu=4 / 3$ for $D=2$ and $\nu \simeq 0.88$ for $D=3$ ). For $D$ $=3$ and by using $t_{0} \simeq 2.0$ and $\nu \simeq 0.88$ the value of $\alpha$ beyond which universility is lost is $\alpha_{c}=1-1 /\left(t_{0}-\nu\right) \simeq 0.107$. Only recently Eq. (5) has been demonstrated to be valid to all orders of a $\epsilon=6-D$ expansion in a renormalization group analysis. ${ }^{59}$

In the original work of Ref. $49, \alpha$ was considered as no more than a parameter of the theory without a justification on microscopic basis. This came later with the RV models and the tunneling-percolation theories. ${ }^{50-52}$ However, before discussing the microscopic aspect, we find it interesting to point out that Eq. (5) predicts that $t$ cannot be lower than $t_{0}$ and it is in principle not bounded above. This is in qualitative agreement with the experimental values of $t$ reported in Fig. 1. Furthermore, Eq. (5) can give us some information on the distribution of $t$ values expected by the theory. In fact, given some normalized distribution function $f(\alpha)$ for the parameter $\alpha$ and for $D=3$, the distribution $N(t)$ of the $t$ values is

$$
\begin{aligned}
N(t)= & \int_{-\infty}^{1} d \alpha f(\alpha) \delta[t-t(\alpha)]=\delta\left(t-t_{0}\right) \int_{-\infty}^{\alpha_{c}} d \alpha f(\alpha) \\
& +\int_{\alpha_{c}}^{1} d \alpha f(\alpha) \delta\left[t-\nu-\frac{1}{1-\alpha}\right] .
\end{aligned}
$$

If we assume that $f(\alpha)$ can be approximated by a constant $f$ for $\alpha_{c} \leqslant \alpha \leqslant 1$, then the above expression reduces to

$$
N(t)=\left(1-\frac{f}{t_{0}-\nu}\right) \delta\left(t-t_{0}\right)+f\left(\frac{1}{t-\nu}\right)^{2} \theta\left(t-t_{0}\right),
$$

which predicts a rapid decay $N(t) \propto(t-\nu)^{-2}$ for $t>t_{0}$. Equation (7) is plotted in Fig. 2 together with the distribution of $t$ values reported in Fig. 1. The distribution $N(t)$ has been renormalized to the number of $t$ values and the constant $f$ has been fixed to reproduce the number of data with $t \geqslant 3.0$. There is an overall qualitative agreement between the distribution of the experimental $t$ values and $N(t)$. In particular, the asymmetry of the distribution and its tail for $t>t_{0} \simeq 2.0$ are well reproduced. Fitting to a power law leads to a decay proportional to $(t-\nu)^{-2.5 \pm 0.4}$ (see inset of Fig. 2) which is in fair agreement with the predicted behavior $(t-\nu)^{-2}$. Due to

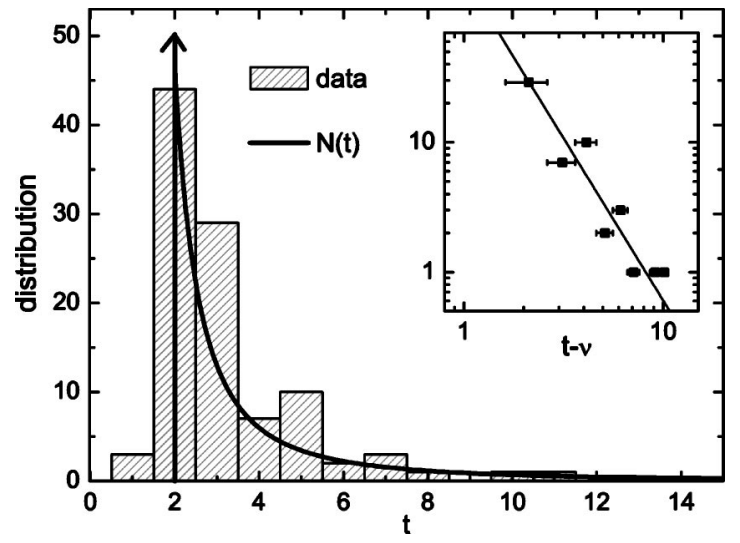

FIG. 2. Distribution of the $t$ values reported in Fig. 1. The solid line is Eq. (7) renormalized in order to reproduce the total number of data. Inset: log-log plot of the distribution with a fit to the power law $a(t-\nu)^{-b}$ with $a=192 \pm 62$ and $b=2.5 \pm 0.4$.

the limited number of $t$ values available to us, the agreement with Eq. (7) could be fortuitous. However, the point here is that the gradual decrease of the number of times high values of $t$ are reported is not necessarily due to "bad fits" to Eq. (1), ${ }^{37,53}$ but it can be, at least qualitatively, explained by the form of Eq. (5).

Let us now discuss the microscopic origin of the exponent $\alpha$. According to the original RV model, ${ }^{50}$ the crucial parameter is the conducting channel width $\delta$ left over from neighboring insulating spheres. For $D=3$, the cross section of the conducting channel has roughly the shape of a triangle and the resulting channel conductance $g$ scales as $g \propto \delta^{3 / 2}$.50 Hence, if $p(\delta)$ is the distribution function of the channel width $\delta$, the distribution $h(g)$ of the conductances reduces to

$$
h(g)=\int d \delta p(\delta) \delta\left(g-g_{0} \delta^{3 / 2}\right),
$$

where $g_{0}$ is a proportionality constant. For random distribution of equally sized spheres, $p(\delta)$ is a constant for $\delta \rightarrow 0$ and Eq. (8) gives $h(g) \propto g^{-1 / 3}$ for $g \rightarrow 0 .^{50} h(g)$ is therefore of the same form of Eq. (4) with $\alpha=1 / 3>\alpha_{c}$ which, according to Eq. (5), predicts a critical exponent $t=\nu+3 / 2 \simeq 2.38$. Despite the fact that this value is only slightly larger than the universal exponent $t_{0} \simeq 2.0$, the RV model has the merit of being a fully microscopic justification of Eq. (4). In order to allow for higher values of $t$, in Ref. 52 it has been proposed to relax the condition that $p(\delta)$ is a constant for $\delta \rightarrow 0$ by introducing the more general condition $p \propto \delta^{-\omega}$ where $\omega<1$. By using Eq. (8) one readily finds that $h(g)$ is again given by Eq. (4) but with $\alpha=\frac{1}{3}+\frac{2}{3} \omega{ }^{52}$ Hence, the critical exponent is $t=t_{0}$ for $\omega<\omega_{c}=\frac{3}{2} \alpha_{c}+\frac{1}{3} \simeq 0.4$ or $t=\nu+\frac{3}{2} /(1-\omega)$ for $\omega>\omega_{c}$, i.e., $t$ is not bounded above. The use of $p \propto \delta^{-\omega}$ has been justified to be a simple ansatz to describe real composites in which correlations between the conducting and insulating phases may yield to deviations from the ideal RV system.

It is important to stress that the RV model of Ref. 50 does not give a breakdown of universality, but rather to a universality class different from that of the standard dc transport percolation on a lattice. In particular, the exponent $t=\nu+\frac{3}{2}$ 
does not depend on the conductivity of the continuum and an applied isotropic strain or pressure, albeit affecting the channel width $\delta$, does not change the relation $g \propto \delta^{3 / 2}$. This should remain true also for the generalized RV theory of Ref. 52 .

Contrary to the RV models, the tunneling-percolation theory of nonuniversality allows for a transport critical exponent dependent of the microscopic conductivites. ${ }^{51}$ In this model, current flows through tunneling processes between neighboring conducting spheres dispersed in an insulating medium and, for sufficiently low concentrations of the conducting spheres, the ensemble of tunneling bonds form a percolating network. The coexistence of tunneling and percolation has been recently settled by experiments probing the local electrical connectivity of various disordered systems, and a recent review on this issue can be found in Ref. 61. In what follows, we consider the situation in which grain charging effects can be neglected with respect to the tunneling processes, as encountered in systems with sufficiently large conducting grains and/or high temperatures. Let us consider then the interparticle tunneling conductance

$$
g=g_{0} e^{-2(r-\Phi) / \xi},
$$

where $g_{0}$ is a constant, $\xi$ is the tunneling factor which is of the order of few nm, $r$ is the distance between the centers of two neighboring spheres of diameter $\Phi(r \geqslant \Phi$ for impenetrable particles). If we denote with $P(r)$ the distribution function of adjacent intersphere distances $r$, the bond conductances are then distributed according to

$$
h(g)=\int_{\Phi}^{\infty} d r P(r) \delta\left[g-g_{0} e^{-2(r-\Phi) / \xi}\right] .
$$

Balberg notices that if $P(r)$ had a slower decay than Eq. (9) for $r \rightarrow \infty$, then $h(g)$ would develop a divergence for $g \rightarrow 0$. For example, by assuming that

$$
P(r)=\frac{e^{-(r-\Phi) /(a-\Phi)}}{a-\Phi},
$$

is a good approximation for the (normalized) distribution of interparticle distances, then Eq. (10) would reduce to

$$
h(g)=\frac{1-\alpha}{g_{0}}\left(\frac{g}{g_{0}}\right)^{-\alpha}
$$

with $\alpha=1-\xi / 2 /(a-\Phi)$, where $a$ is the mean distance between neighboring particles. For $\xi / 2 /(a-\Phi) \leqslant 1-\alpha_{c} \simeq 0.9$, transport becomes nonuniversal with $t=\nu+2[(a-\Phi) / \xi] .{ }^{51} \mathrm{It}$ is important to stress that here nonuniversality is not driven by geometrical factors as in the RV model, but rather by physical parameters such as $\xi$ and $a$. These can be different depending on the composite and can be modified by a suitable external perturbation. In fact, in the case of an applied pressure or strain, the mean tunneling distance $a$ would change leading to a modification of the critical exponent $t$. As already pointed out in the introduction, the detection of such an effect would be a direct signature of a tunnelingpercolation-like mechanism of transport nonuniversality.
The tunneling-percolation theory in its original formulation relies on Eq. (11) which should be regarded as a phenomenological model of the distribution function of interparticle distances. Recently, however, a microscopic derivation of Eq. (11) has been formulated for a bond-percolation regular network in which the bonds have probability $p$ of being occupied by a string of $n$ nonoverlapping spheres. ${ }^{62}$ In this case, in fact, Eq. (11) is the exact distribution function for spheres placed in a one-dimensional channel, ${ }^{63}$ and the resulting bond conductance distribution is proportional to $g^{-\alpha_{n}}$ where $\alpha_{n}=1-\xi / 2 /\left(a_{n}-\Phi\right)$ and $a_{n}$ is the mean interparticle distance for a bond occupied by $n$ spheres. Despite its oversimplification, this construction shows, however, that the tunneling-percolation mechanism of nonuniversality can be justified by a fully defined model, without need of phenomenological forms of $P(r)$.

\section{THEORY OF PIEZORESISTIVITY}

The sensitivity of the tunneling-percolation mechanism of nonuniversality to variations of the mean tunneling distance can be exploited by imposing a volume compression or expansion to the system. As we discuss below, under these circumstances the relative change of resistivity, i.e., the piezoresistive response, changes dramatically depending on whether the dc exponent is universal $\left(t=t_{0}\right)$ or instead it is driven away from universality by the tunneling-percolation mechanism.

Let us consider the rather general situation in which a parallelepiped with dimensions $L_{x}, L_{y}$, and $L_{z}$ is subjected to a deformation along its main axes $x, y$, and $z$. The initial volume $V=L_{x} L_{y} L_{z}$ changes to $V(1+\phi)$, where $\phi=\varepsilon_{x}+\varepsilon_{y}+\varepsilon_{z}$ is the volume dilatation and $\varepsilon_{i}=\delta L_{i} / L_{i}$ are the principal strains along $\varepsilon_{i}$ with $i=x, y, z$. In the absence of strain, we assume that the conductivity $\Sigma$ of the parallelepiped is isotropic, so that the conductance $G_{i}$ measured along the $i$ axis is $G_{i}=\Sigma L_{j} L_{k} / L_{i}$. For small $\varepsilon_{i} \neq 0(i=x, y, z)$, the conductance variation $\delta G_{i}$ is therefore

$$
\frac{\delta G_{i}}{G_{i}}=\frac{\delta \Sigma_{i}}{\Sigma}-\varepsilon_{i}+\varepsilon_{j}+\varepsilon_{k},
$$

where

$$
\frac{\delta \Sigma_{i}}{\Sigma}=-\Gamma_{\|} \varepsilon_{i}-\Gamma_{\perp}\left(\varepsilon_{j}+\varepsilon_{k}\right)
$$

are the relative variation of the conductivity along the $i$ $=x, y, z$ directions. The coefficients $\Gamma_{\|}$and $\Gamma_{\perp}$ are the longitudinal and transverse piezoresistive factors defined as

$$
\begin{gathered}
\Gamma_{\|}=-\frac{d \ln \left(\sum_{i}\right)}{d \varepsilon_{i}}=\frac{d \ln \left(\rho_{i}\right)}{d \varepsilon_{i}}, \\
\Gamma_{\perp}=-\frac{d \ln \left(\sum_{j}\right)}{d \varepsilon_{i}}=\frac{d \ln \left(\rho_{j}\right)}{d \varepsilon_{i}}(i \neq j),
\end{gathered}
$$

where $\rho_{i}=\Sigma_{i}^{-1}$ is the resistivity along the $i$ axis and $\ln$ is the natural logarithm. The distinction between longitudinal $\Gamma_{\|}$ and transverse $\Gamma_{\perp}$ piezoresistive responses is important 
whenever the values of the strains $\varepsilon_{i}$ depend on the direction, as it is encountered when the sample is subjected to uniaxial distortions as those induced in cantilever beam experiments (see next section).

In what follows, we are mainly interested in the isotropic (or hydrostatic) piezoresistive factor $\Gamma$ defined as the resistivity change induced by the isotropic strain field $\varepsilon_{i}=\varepsilon$ for all $i=x, y, z$ :

$$
\Gamma=-\frac{d \ln (\Sigma)}{d \varepsilon}=\frac{d \ln (\rho)}{d \varepsilon},
$$

which can be obtained by applying a hydrostatic pressure to the parallelepiped. However, $\Gamma$ can also be obtained by setting $\varepsilon_{i}=\varepsilon$ in Eq. (14) yielding

$$
\Gamma=\Gamma_{\|}+2 \Gamma_{\perp},
$$

which is a useful relation when the experimental setup does not permit to apply an isotropic strain field.

Let us now study how the tunneling-percolation theory of nonuniversality affect the piezoresistive factor $\Gamma$. To this end, we assume that a cubic bond-percolation network is embedded in a homogeneous elastic medium and that the elastic coefficients of the network and the medium are equal. Under an isotropic strain field $\varepsilon_{i}=\varepsilon(i=x, y, z)$ the mean tunneling distance $a$ changes to $a(1+\varepsilon)$ independently of the bond orientation. Hence, by assuming for simplicity that $\Phi \rightarrow \Phi(1$ $+\varepsilon)$, the tunneling parameter $\alpha=1-\xi / 2 /(a-\Phi)$ entering Eq. (5) becomes $\alpha \rightarrow \alpha+(1-\alpha) \varepsilon$ for $\varepsilon \ll 1$. According to the discussion of Sec. II and to Eqs. (3), (5), and (17) close to the percolation threshold $p_{c}$, the piezoresistive factor behaves therefore as

$$
\Gamma= \begin{cases}\Gamma_{0}, & \alpha \leqslant \alpha_{c}, \\ \Gamma_{0}-\frac{d t}{d \varepsilon} \ln \left(p-p_{c}\right), & \alpha>\alpha_{c},\end{cases}
$$

where

$$
\begin{gathered}
\Gamma_{0}=-\frac{d \ln \left(\Sigma_{0}\right)}{d \varepsilon}=-(1-\alpha) \frac{d \ln \left(\Sigma_{0}\right)}{d \alpha}, \\
\frac{d t}{d \varepsilon}=\frac{1}{1-\alpha}=2(a-\Phi) / \xi,
\end{gathered}
$$

where we have used $d \alpha / d \varepsilon=(1-\alpha)$. The tunneling distance dependence of the dc transport exponent is therefore reflected in a logarithmic divergence of $\Gamma$ as $p \rightarrow p_{c}$. Instead, when $\alpha \leqslant \alpha_{c}$, the dc exponent remains equal to $t_{0}$ also when $\varepsilon \neq 0$ and the resulting piezoresistive factor is simply equal to $\Gamma=\Gamma_{0}$, independently of the bond probability $p$.

It is worth to note that, as shown in Ref. 64 , contrary to $\Gamma$, the breakdown of universality has no effect on the piezoresistive anisotropy defined as $\chi=\left(\Gamma_{\|}-\Gamma_{\perp}\right) / \Gamma_{\|}$which behaves as $\chi \propto\left(p-p_{c}\right)^{\lambda}$ where the critical exponent $\lambda$ is independent of $\alpha$ also when $\alpha>\alpha_{c}{ }^{64}$ Equation (19) is an exact result as long as we are concerned with the $p-p_{c}$ dependence close to the percolation threshold. However, in addition to the prefactor of the logarithm, $\Gamma$ depends also on the tunneling parameter $\alpha$ through the term $\Gamma_{0}$. This dependence is far from trivial. In fact, consider a tensile strain $(\varepsilon>0)$ which enhances the bond tunneling resistances leading to an overall enhancement of the sample resistivity. In this case, $\Gamma$ $=d \ln (\rho) / d \varepsilon$ must be strictly positive. This means that $\Gamma_{0}$ $>0$ when $\alpha \leqslant \alpha_{c}$ while, from Eq. (19), when $\alpha>\alpha_{c} \Gamma_{0}$ does not need to be positive to ensure $\Gamma>0$ because of the presence of the logarithmic divergence. In the next subsections we provide evidence that indeed $\Gamma_{0}$ changes sign in passing from $\alpha \leqslant \alpha_{c}$ to $\alpha>\alpha_{c}$ by showing that both the effective medium approximation and numerical calculations on cubic lattices give negative values of $\Gamma_{0}$ for nonuniversal dc transport. Together with the logarithmic divergence of $\Gamma$ for $p$ $\rightarrow p_{c}$, a negative value of $\Gamma_{0}$ would be an additional signature of a tunneling-percolation mechanism of nonuniversality.

\section{A. Effective medium approximation}

In the effective medium approximation (EMA) the conductivity $\Sigma=\bar{g} / \ell$ of a bond percolation cubic lattice (with bond length $\ell$ ) is obtained by the solution of the following integral equation (see, for example, Ref. 2):

$$
\int_{0}^{\infty} d g \rho(g) \frac{\bar{g}-g}{g+2 \bar{g}}=0,
$$

where $\rho(g)$ is the distribution function of the bond conductances $g$ given in Eq. (2). Close to the percolation threshold $p_{c}\left(p_{c}=1 / 3\right.$ in EMA $)$ and by using the tunneling-percolation distribution of Eq. (12) with $g_{0}=1$, the above expression reduces to

$$
(1-\alpha) \bar{g} \int_{0}^{1} d g \frac{g^{-\alpha}}{g+2 \bar{g}}=\frac{3}{2}\left(p-p_{c}\right) .
$$

It is clear that for $\bar{g} \rightarrow 0$ the integral in Eq. (23) remains finite as long as $\alpha<0$, while for $\alpha>0$ it diverges as $\bar{g}^{-\alpha}$. Hence, for $p \rightarrow p_{c}$ the conductivity $\Sigma$ follows the power-law behavior of Eq. (3) with $t=1$ for $\alpha<0$ and $t=1 /(1-\alpha)$ for $\alpha>0$. The corresponding prefactor $\Sigma_{0}$ can be evaluated explicitly:

$$
\begin{gathered}
\Sigma_{0}(\alpha<0)=\frac{3}{2} \frac{\alpha}{1-\alpha}, \\
\Sigma_{0}(\alpha>0)=\left[\frac{3}{2^{1-\alpha} \gamma(\alpha) \gamma(2-\alpha)}\right]^{1 /(1-\alpha)},
\end{gathered}
$$

where $\gamma$ is the Euler gamma function. Note that the above expressions give $\Sigma_{0}(\alpha \rightarrow 0)=0$, which is a result due to enforcing $\Sigma$ to behave as Eq. (3). Actually, at $\alpha=0$ and for $\bar{g}$ $\ll 1$ Eq. (23) reduces to $-\bar{g} \ln (2 \bar{g})=\frac{3}{2}\left(p-p_{c}\right)$ which leads to logarithmic corrections in the $p-p_{c}$ dependence of $\bar{g}$. For $\alpha \neq 0$, the logarithmic corrections are not important only in a region around $p=p_{c}$ which shrinks to zero as $\alpha \rightarrow 0$.

$\Sigma_{0}(\alpha)$ is plotted in Fig. 3 as a function of $\alpha$. For $\alpha<0$ $(\alpha>0), \Sigma_{0}(\alpha)$ is a decreasing (increasing) function of $\alpha$. Hence, according to the second equality of Eq. (20), $\Gamma_{0}$ is expected to be positive for $\alpha<0$ and negative for $\alpha>0$. This is confirmed in the inset of Fig. 3 where $\Gamma_{0}$ is plotted as a function of $\alpha$. Note that as $\alpha \rightarrow 1, \Gamma_{0}$ goes to $-\infty$. In fact, from Eq. (25), in this regime $\Sigma_{0}(\alpha) \simeq \frac{1}{2} 3^{1 /(1-\alpha)}=\frac{1}{2} 3^{t}$ which implies that 


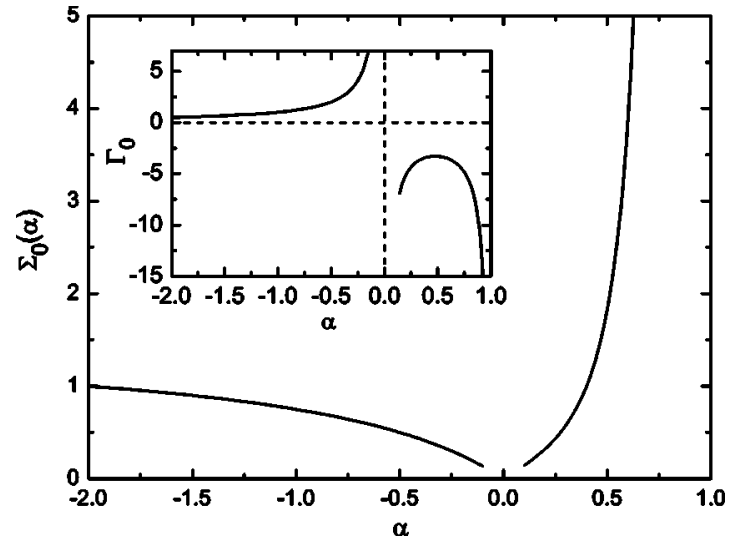

FIG. 3. Prefactor $\Sigma_{0}$ of Eq. (3) as a function of the tunneling parameter $\alpha$. Note that $\Sigma_{0}$ is a decreasing (increasing) function of $\alpha$ for $\alpha<0(\alpha>0)$. Inset: the $p$ independent contribution $\Gamma_{0}$ to the piezoresistive factor $\Gamma$. Note that $\Sigma_{0}$ and $\Gamma_{0}$ are not plotted for small values of $\alpha$ because in this region the logarithmic corrections to $\Sigma$ calculated within EMA change the simple power-law behavior of Eq. (3).

$$
\Gamma_{0} \simeq-\ln (3) \frac{d t}{d \varepsilon}
$$

\section{B. Monte Carlo calculations on cubic lattices}

To evaluate the $\alpha$ dependence of the prefactor $\Sigma_{0}$ of Eq. (3) we have used the transfer-matrix method applied to a cubic lattice on $N-1$ sites in the $z$ direction, $N$ sites along $y$, and $\mathcal{L}$ along the $x$ direction. ${ }^{65}$ Periodic boundary conditions are used in the $y$ direction while a unitary voltage is applied to the top plane, and the bottom plane is grounded to zero. For sufficiently large $\mathcal{L}(\mathcal{L} \gg N)$ this method permits to calculate the conductivity $\Sigma_{N}$ per unit length of a cubic lattice of linear size $N$. The transfer matrix algorithm is particularly efficient at the bond percolation threshold $p_{c} \simeq 0.2488126$, and it is usually used in connection with finite size scaling analysis of $\Sigma_{N}$ to extract highly accurate values of the dc exponent $t .^{4}$

In performing the calculations we have considered the following geometries: $N=6\left(\mathcal{L}=5 \times 10^{7}\right), N=8\left(\mathcal{L}=2 \times 10^{7}\right)$, $N=10\left(\mathcal{L}=1 \times 10^{7}\right), \quad N=12\left(\mathcal{L}=8 \times 10^{6}\right), \quad N=14\left(\mathcal{L}=4 \times 10^{6}\right)$, and $N=16\left(\mathcal{L}=2 \times 10^{6}\right)$. From Eq. (3), the resulting conductivity $\Sigma_{N}$ for finite $N$ at $p=p_{c}$ can be written as

$$
\Sigma_{N}=\Sigma_{0}(\alpha)\left[p_{c}-p_{c}(N)\right]^{t(\alpha)}
$$

where we have explicitly written the $\alpha$ dependence of the prefactor and of the exponent. In the above expression, $p_{c}(N)$ is the percolation threshold of a finite system of linear size $N$. Only at $N \rightarrow \infty, p_{c}(N)$ coincides with the percolation threshold $p_{c}$ of an infinite system, while for finite values of $N$ the two quantities are related via a finite size scaling relation of the type ${ }^{66}$

$$
p_{c}-p_{c}(N) \simeq A N^{-1 / \nu}\left(1+B N^{-\omega}\right),
$$

where $\nu \simeq 0.88$ is the correlation length exponent, $A$ and $B$ are constant, and $\omega$ is the first scaling correction exponent.

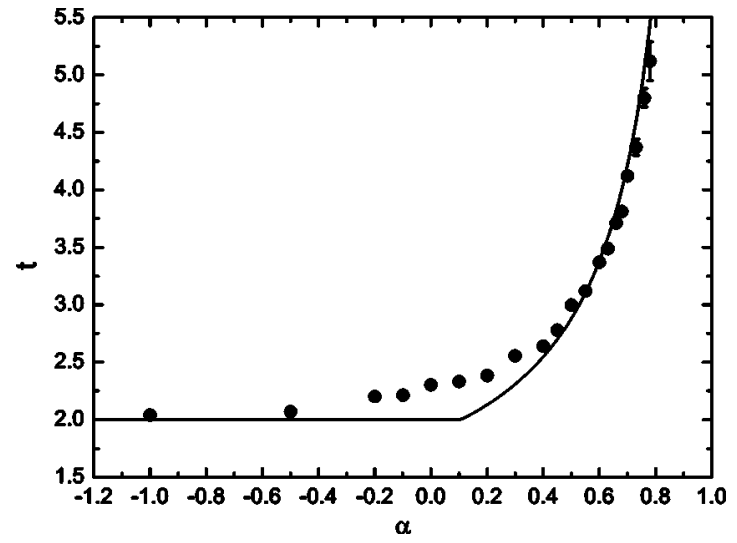

FIG. 4. dc transport exponent $t$ calculated from the transfermatrix method as a function of the parameter $\alpha$. The solid line is the exact result (5).

All the constants appearing in Eq. (28) depend solely on the connectivity of the network, and are therefore independent of the tunneling factor $\alpha$. By inserting Eq. (28) in Eq. (27), the conductivity reduces to

$$
\Sigma_{N} \simeq \bar{\Sigma}_{0}(\alpha) N^{-t(\alpha) / \nu}\left(1+B N^{-\omega}\right)^{t(\alpha)},
$$

where

$$
\bar{\Sigma}_{0}(\alpha)=\Sigma_{0}(\alpha) A^{t(\alpha)} .
$$

Our strategy to calculate $\Sigma_{0}(\alpha)$ is the following. We first fit our numerical data of $\Sigma_{N}$ with Eq. (29) by setting $\omega$ fixed. In this way we obtain the exponent $t(\alpha)$ and the prefactor $\bar{\Sigma}_{0}(\alpha)$. We repeat this procedure for various values of $\alpha$ ranging from $\alpha \simeq 1$ down to $\alpha=-\infty$ which corresponds to the Dirac delta distribution function $h(-\infty)=\delta(g-1)$. In this limit, $\Sigma_{0}(-\infty)$ is known with a rather good accuracy, permitting us to calculate from Eq. (30) the value of the constant $A$. In this way we finally obtain $\Sigma_{0}(\alpha)=\bar{\Sigma}_{0}(\alpha) / A^{t(\alpha)}$ by using the values of the exponent calculated before.

In Fig. 4 we plot the values of the exponent $t$ as a function of $\alpha$ obtained by setting $\omega=1$ in Eq. (29). We have checked that this choice for $\omega$ produced the best overall agreement with the exact result (5) shown in Fig. 4 by the solid line. In accord with the $\alpha$ independence of $B$, we noticed little deviations from $B \simeq 0.7$ in the whole range of $\alpha$. The agreement between the calculated exponent and Eq. (5) is very good far away from $\alpha=\alpha_{c} \simeq 0.107$. In the vicinity of $\alpha_{c}$ the competition between two different fixed points leads to a less good agreement, as already noticed in previous works. ${ }^{67}$

In Fig. 5 we report the calculated values of the prefactor $\bar{\Sigma}_{0}(\alpha)$ of the finite size scaling relation (29). Note that the overall dependence of $\bar{\Sigma}_{0}(\alpha)$ upon $\alpha$ resembles that of Fig. 3, although the presence of $A^{t(\alpha)}$ certainly affects the $\alpha>\alpha_{c}$ region. In the inset of Fig. 5 we have plotted the behavior of $\bar{\Sigma}_{0}(\alpha)$ as $\alpha \rightarrow-\infty$. In this regime, the exponent is universal $\left(t=t_{0} \simeq 2\right)$ and the whole $\alpha$ dependence is contained in the conductivity prefactor $\Sigma_{0}(\alpha)$. From Ref. 5 we know that $\Sigma_{0}(-\infty) \simeq 0.4$, while we have obtained $\bar{\Sigma}_{0}(-\infty) \simeq 0.96$. Hence from Eq. (30) we have $A \simeq(0.96 / 0.4)^{1 / 2} \simeq 1.55$. 


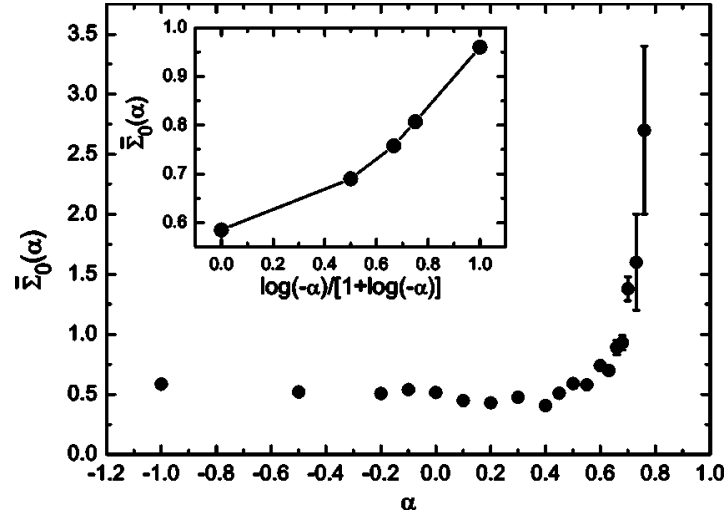

FIG. 5. Prefactor $\bar{\Sigma}_{0}(\alpha)$ of the finite size scaling relation (29) as a function of $\alpha$. Inset: behavior of $\bar{\Sigma}_{0}(\alpha)$ as a function of $\log (-\alpha) /[1+\log (-\alpha)]$ (where $\log$ is the logarithm to base 10) for $\alpha=-1,-10,-100,-1000$, and $\alpha=-\infty$. This latter case corresponds to a Dirac delta distribution function $h(g)=\delta(g-1)$ for which we obtain $\bar{\Sigma}_{0}(-\infty)=0.960 \pm 0.007$.

Our final results for $\Sigma_{0}(\alpha)=\bar{\Sigma}_{0}(\alpha) / 1.55^{t(\alpha)}$ are plotted in Fig. 6, where the $t(\alpha)$ values are those plotted in Fig. 4. As for the EMA case, $\Sigma_{0}(\alpha)$ decreases for $\alpha$ sufficiently smaller than $\alpha_{c} \simeq 0.107$ while it increases for $\alpha>\alpha_{c}$. Hence, also our Monte Carlo calculations confirm that the $p$-independent part $\Gamma_{0}$ of the piezoresistive response is positive or negative, depending whether $\alpha$ is less than or larger than $\alpha_{c}$, respectively. In the inset of Fig. 5 we report a semilogarithmic plot of $\Sigma_{0}(\alpha)$ as a function of the dc exponent $t$. For high values of $t$, the data are reasonably well fitted by a relation of the form $\Sigma_{0}(\alpha)=a b^{t}$ with $a=0.018 \pm 0.004$ and $b=1.9 \pm 0.1$ (solid line in the inset of Fig. 6. Hence,

$$
\Gamma_{0}=-\ln (b) \frac{d t}{d \varepsilon} \simeq-0.6 \frac{d t}{d \varepsilon}
$$

confirming the asymptotic formula (26) obtained within EMA.

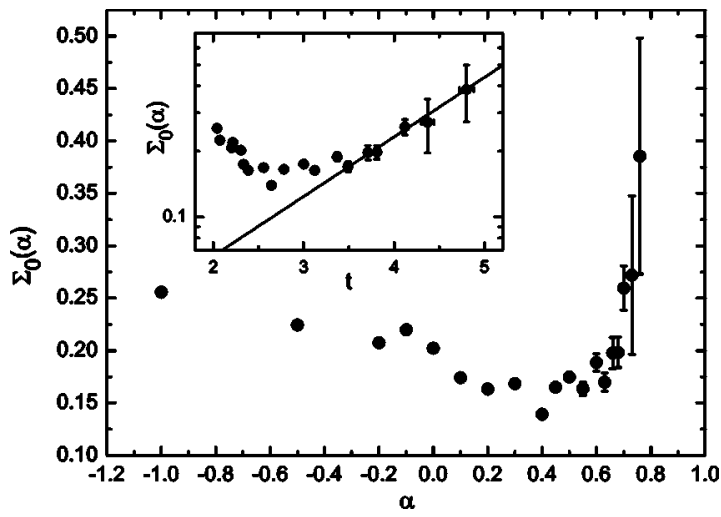

FIG. 6. Prefactor $\Sigma_{0}(\alpha)$ of the conductivity obtained from Eq. (30) with $A=1.55$ and $t$ from Fig. 4. Inset: semilogarithmic plot of $\Sigma_{0}(\alpha)$ as a function of the dc exponent $t$. Solid line is a fit with $\Sigma_{0}(\alpha)=a b^{t}$ with $a=0.018 \pm 0.004$ and $b=1.9 \pm 0.1$.

\section{EXPERIMENT}

In this section we describe our experiments aimed to investigate the piezoresistive response of disordered conductor-insulator composites made of conducting $\mathrm{RuO}_{2}$ particles embedded in an insulating glass. As shown in Fig. 1 , this kind of TFR displays both universal $\left(t \simeq t_{0}\right)$ and nonuniversal $\left(t>t_{0}\right)$ behaviors of transport, although the factors responsible for such changes have not yet been identified. In addition, transport in such kind of materials is known to be governed by electron tunneling through the glassy film separating two neighboring conducting particles. ${ }^{55-57}$ Hence, $\mathrm{RuO}_{2}$-based TFRs are ideal systems to test whether a tunneling-percolation mechanism of transport nonuniversality sets in.

Our samples were prepared starting with a glass frit with the following composition: $\mathrm{PbO}$ (75\% wt.), $\mathrm{B}_{2} \mathrm{O}_{3}$ (10\% wt.), $\mathrm{SiO}_{2}$ (15\% wt.). In order to avoid crystallisation, $2 \%$ wt. of $\mathrm{Al}_{2} \mathrm{O}_{3}$ was added to the glass powder. After milling, the glass powder presented an average grain size of about $3 \mu \mathrm{m}$, as measured from laser diffraction analysis. Thermogravimetric measurements showed negligible loss in weight, indicating glass stability and no $\mathrm{PbO}$ evaporation during firing up to $800{ }^{\circ} \mathrm{C}$, and differential scanning calorimetry measurements indicated a softening temperature of about $460{ }^{\circ} \mathrm{C}$. For the conductive phase we used two different $\mathrm{RuO}_{2}$ powders with nominal grain sizes of $400 \mathrm{~nm}$ (series $A$ ) and $40 \mathrm{~nm}$ (series $B$ ). Transmission electron microscope analysis confirmed that the finer powder was made of nearly spherical particles with a diameter of about $40 \mathrm{~nm}$, while the coarser powder had more dispersed grain sizes (100 $\mathrm{nm}$ mean) with less regular shape.

TFRs were then prepared by mixing several weight fractions of the two series of $\mathrm{RuO}_{2}$ powder with the glass particles. An organic vehicle made of terpineol and ethyl cellulose was added in a quantity of about $30 \%$ of the total weight of the $\mathrm{RuO}_{2}$-glass mixture. The so-obtained pastes were screen printed on $96 \%$ alumina substrates on prefired gold terminations. For the conductance measurements, eight resistors $1.5 \mathrm{~mm}$ wide and different lengths ranging from $0.3 \mathrm{up}$ to $5 \mathrm{~mm}$ were printed on the same substrate. The resistors were then treated with a thermal cycle consisting of a drying phase $\left(10 \mathrm{~min}\right.$ at $\left.150{ }^{\circ} \mathrm{C}\right)$ followed by a plateau, reached at a rate of $20^{\circ} \mathrm{C} / \mathrm{min}$, of $15 \mathrm{~min}$ at various firing temperatures $T_{f}$ (see below and Table I). After firing, the thickness of the films was about $10 \mu \mathrm{m}$.

In Figs. 7 and 8 we show scanning electron microscope (SEM) images of the surfaces of $A$ and $B$ series, respectively, with $\mathrm{RuO}_{2}$ volume concentration $x=0.08$. The firing temperature $T_{f}$ is $T_{f}=525^{\circ} \mathrm{C}$ and $T_{f}=600{ }^{\circ} \mathrm{C}$ for the $A$ series [Figs. 7(a) and 7(b), respectively], while $T_{f}=550{ }^{\circ} \mathrm{C}$ and $T_{f}$ $=600{ }^{\circ} \mathrm{C}$ for the $B$ series [Figs. 8(a) and 8(b), respectively]. In the images, dark areas indicate highly conducting regions rich of $\mathrm{RuO}_{2}$ clusters, while the white zones are instead made of insulating glass. The grey regions surrounding the $\mathrm{RuO}_{2}$ clusters indicate that some conduction is present, although much lower than the dark areas, which we ascribe to finer $\mathrm{RuO}_{2}$ particles dispersed in the glass. ${ }^{68}$ At the length scale shown in the figures the conducting and insulating phases are not dispersed homogeneously, with the $\mathrm{RuO}_{2}$ 
TABLE I. Label legend of the various samples used in this work with fitting parameters of Eqs. (1) and (34).

\begin{tabular}{|c|c|c|c|c|c|c|c|}
\hline Label & $\mathrm{RuO}_{2}$ grain size & firing temperature $T_{f}$ & $x_{c}$ & $\ln \left(\sigma_{0} \Omega \mathrm{m}\right)$ & $t$ & $\Gamma_{0}$ & $d t / d \varepsilon$ \\
\hline$A 1$ & $400 \mathrm{~nm}$ & $525^{\circ} \mathrm{C}$ & 0.0745 & $11.1 \pm 0.3$ & $2.15 \pm 0.06$ & $16.5 \pm 4.5$ & $-0.6 \pm 1.2$ \\
\hline$A 2$ & $400 \mathrm{~nm}$ & $600^{\circ} \mathrm{C}$ & 0.0670 & $14.2 \pm 0.2$ & $3.84 \pm 0.06$ & $-26.4 \pm 4.8$ & $16.2 \pm 1.5$ \\
\hline$B 1$ & $40 \mathrm{~nm}$ & $550^{\circ} \mathrm{C}$ & 0.0626 & $14.3 \pm 0.5$ & $3.17 \pm 0.16$ & $-45.9 \pm 9$ & $26.1 \pm 2.7$ \\
\hline$B 2$ & $40 \mathrm{~nm}$ & $600^{\circ} \mathrm{C}$ & 0.0525 & $13.7 \pm 0.7$ & $3.15 \pm 0.17$ & $-57.9 \pm 7.2$ & $33.0 \pm 2.1$ \\
\hline
\end{tabular}

clusters segregated between large glassy regions of few micrometers in size. This segregation effect in TFRs is well known and it is due to the large difference in size between the fine conducting particles and the much coarser glassy grains employed in the preparation of the resistors. ${ }^{27}$ Concerning the effect of the firing temperature, it is interesting to note that for the $B$ series there is not much qualitative difference in the microstructure between $T_{f}=550{ }^{\circ} \mathrm{C}$ [Fig. 8(a)] and $T_{f}=600{ }^{\circ} \mathrm{C}$ [Fig. 8(b)], while for the $A$ series it appears that the conducting phase is more clustered at low firing temperature [Fig. 7(a)] than at high $T_{f}[$ Fig. 7(b)], where the appearance of grey regions indicate larger $\mathrm{RuO}_{2}$ dispersion in the glass.

In Fig. 9 we report the room-temperature conductivity $\sigma$ measured for four different series of TFRs (see Table I) as functions of the $\mathrm{RuO}_{2}$ volume concentration $x$. As shown in
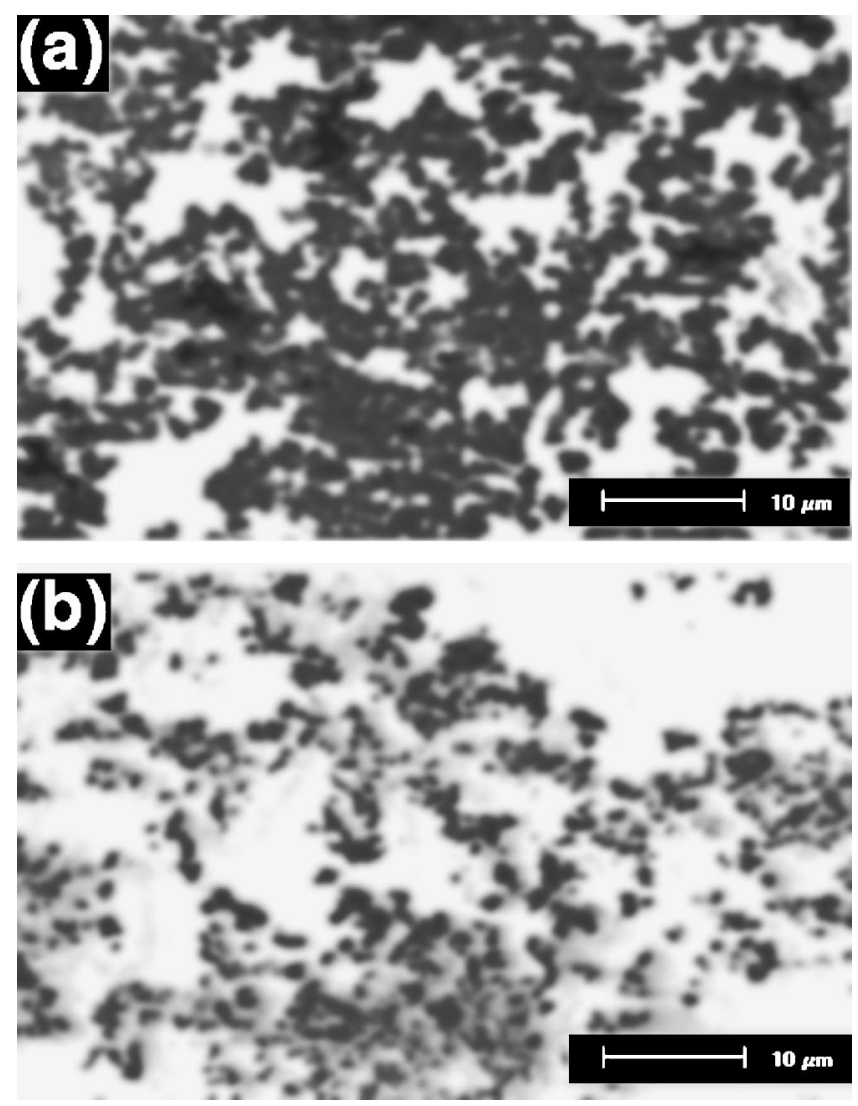

FIG. 7. SEM images of the surface of the $A$ series with $\mathrm{RuO}_{2}$ volume fraction $x=0.08$ and nominal $\mathrm{RuO}_{2}$ grain size of $400 \mathrm{~nm}$ (see text) for different firing temperatures $T_{f}$. (a) $T_{f}=525^{\circ} \mathrm{C}$; (b): $T_{f}=600{ }^{\circ} \mathrm{C}$.
Fig. 9(a), $\sigma$ vanishes at rather small values of $x$, as expected when the mean grain size of the conducting phase $(40 \mathrm{~nm}$ and $<400 \mathrm{~nm}$ ) is much smaller than that of the glass $(1-5 \mu \mathrm{m}) .{ }^{69}$ The same data are replotted in the $\ln -\ln$ plot of Fig. 9(b) together with the corresponding fits to Eq. (1) (solid lines) and the best-fit parameters $\sigma_{0}, x_{c}$, and $t$ are reported in Table I. As is clearly shown, our conductivity data follow the power-law behavior of Eq. (1) with exponent $t$ close to the universal value $t_{0} \simeq 2.0$ for the $A 1$ series $(t=2.15 \pm 0.06)$ or markedly nonuniversal as for the $A 2$ case which displays $t$ $=3.84 \pm 0.06$. The $B 1$ and $B 2$ series have nearly equal values of $t(t \simeq 3.16)$ falling in between those of the $A 1$ and $A 2$ series.

It is tempting to interpret the different transport behaviors of the $A 1$ and $A 2$ series by referring to the microstructures reported in Fig. 7. It appears that universal behavior is found
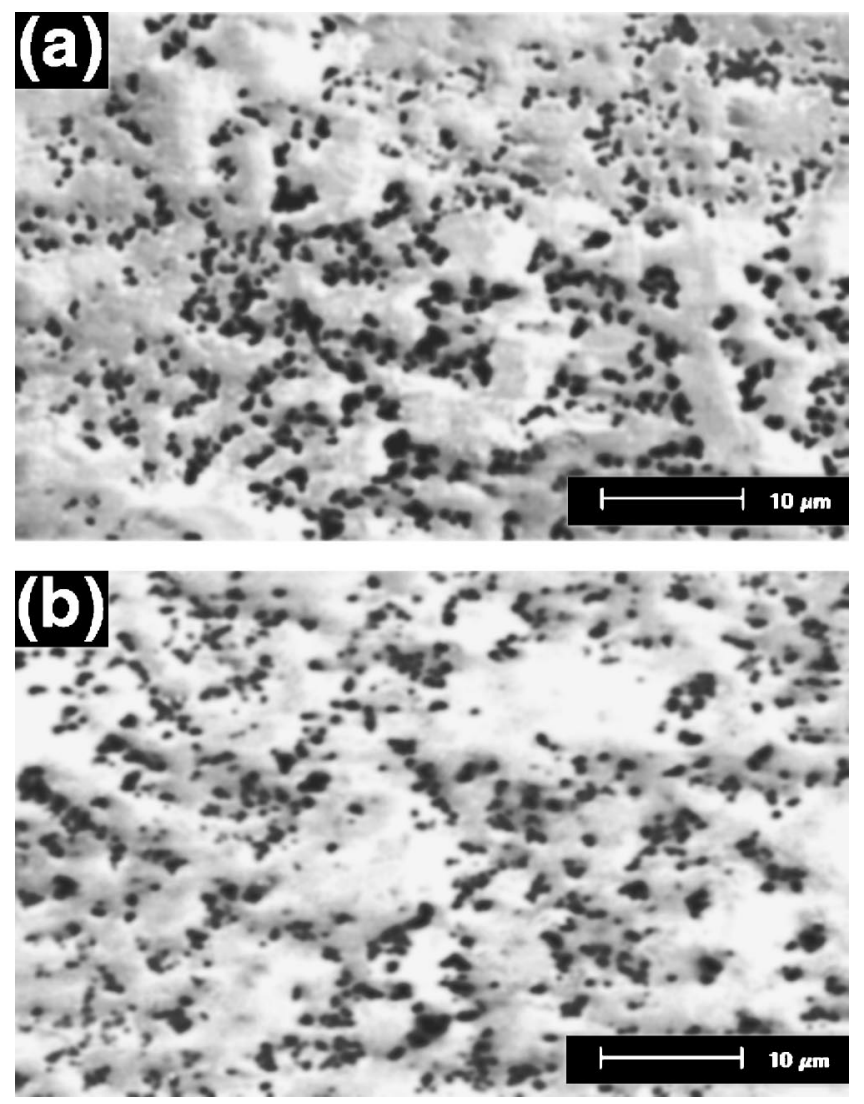

FIG. 8. SEM images of the surface of the $B$ series with $\mathrm{RuO}_{2}$ volume fraction $x=0.08$ and nominal $\mathrm{RuO}_{2}$ grain size of $40 \mathrm{~nm}$ (see text) for different firing temperatures $T_{f}$. (a) $T_{f}=550{ }^{\circ} \mathrm{C}$; (b) $T_{f}$ $=600{ }^{\circ} \mathrm{C}$. 

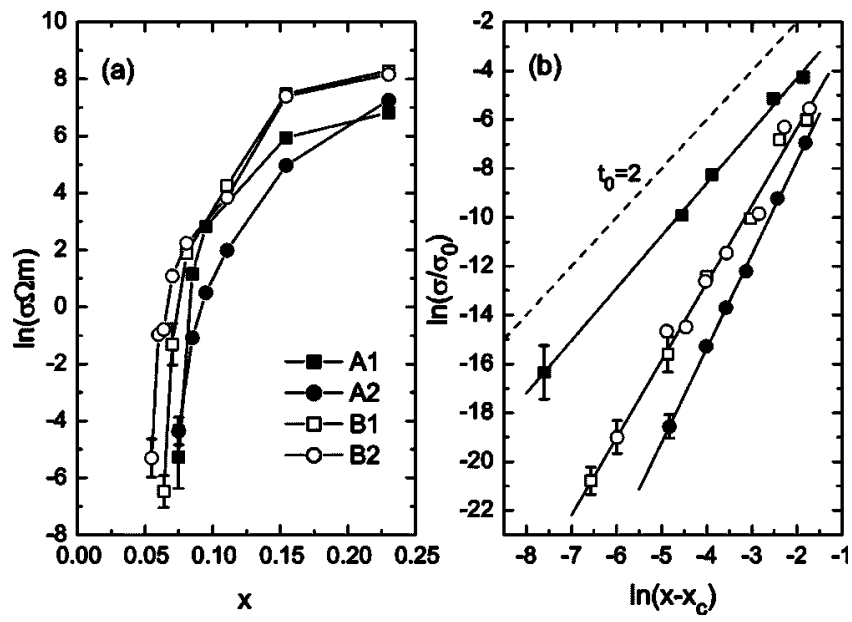

FIG. 9. (a) Conductivity $\sigma$ as a function of $\mathrm{RuO}_{2}$ volume concentration $x$ for four different series of TFRs. (b) ln-ln plot of the same data of (a) with fits to Eq. (1) shown by solid lines. The dashed line has slope $t_{0}=2$ corresponding to universal behavior of transport. The prefactor $\rho_{0}$, critical concentration $x_{c}$ and transport exponent $t$ values obtained by the fits are reported in Table I.

for the more clustered samples [Fig. 7(a)] while the nonuniversal behavior is observed when the conducting phase is more dispersed in the glass [Fig. 7(b)]. This interpretation is coherent with the nonuniversality of both $B 1$ and $B 2$ series, which indeed display a large amount of $\mathrm{RuO}_{2}$ dispersion evidenced by the grey regions in Figs. 8(a) and 8(b). As discussed in Sec. II, the microstructure has a primary role for the onset of nonuniversality. This is certainly true for the tunneling-percolation model in which the microstructure governs the tunneling distribution function. In this respect, the SEM images reported in Figs. 7 and 8 may suggest that for the A1 series [Fig. 7(a)], since the $\mathrm{RuO}_{2}$ grains are less dispersed, the tunneling distribution function is much narrower than those of the other series.

To measure the piezoresistive response, four resistors for each series and with equal $\mathrm{RuO}_{2}$ content were screen printed in a Wheatstone bridge arrangement on the top of alumina cantilever bars $51 \mathrm{~mm}$ long, $b=5 \mathrm{~mm}$ large, and $h$ $=0.63 \mathrm{~mm}$ thick. The thermal treatment was the same as for the samples used for the conductivity measurements. The cantilever was clamped at one end and different weights were applied at the opposite end. The resulting substrate strain $\varepsilon$ along the main cantilever axis can be deduced from the relation $\varepsilon=6 \mathrm{Mgd} /\left(E b h^{2}\right)$, where $d$ is the distance between the resistor and the point of applied force, $E$ $=332.6 \mathrm{GPa}$ is the reduced $\mathrm{Al}_{2} \mathrm{O}_{3}$ Young modulus, $g$ is the gravitational acceleration, and $M$ is the value of the applied weight. By fixing the main cantilever axis parallel to the $x$ direction, then in plain strain approximation the strain field transferred to the resistors is $\varepsilon_{x}=\varepsilon, \varepsilon_{y}=0$, and $\varepsilon_{z}=-\nu /(1$ $-\nu) \varepsilon$, where $\nu=0.22$ is the Poisson ratio of $96 \% \mathrm{Al}_{2} \mathrm{O}_{3}$. Two different cantilevers were used for the measurements of the longitudinal and transverse piezoresistive signals obtained by recording the conductivity changes along the $x$ and $y$ directions, respectively. Then, according to Eq. (14):

$$
\frac{\delta \sigma_{x}}{\sigma}=-\left(\Gamma_{\|}-\Gamma_{\perp} \frac{\nu}{1-\nu}\right) \varepsilon,
$$
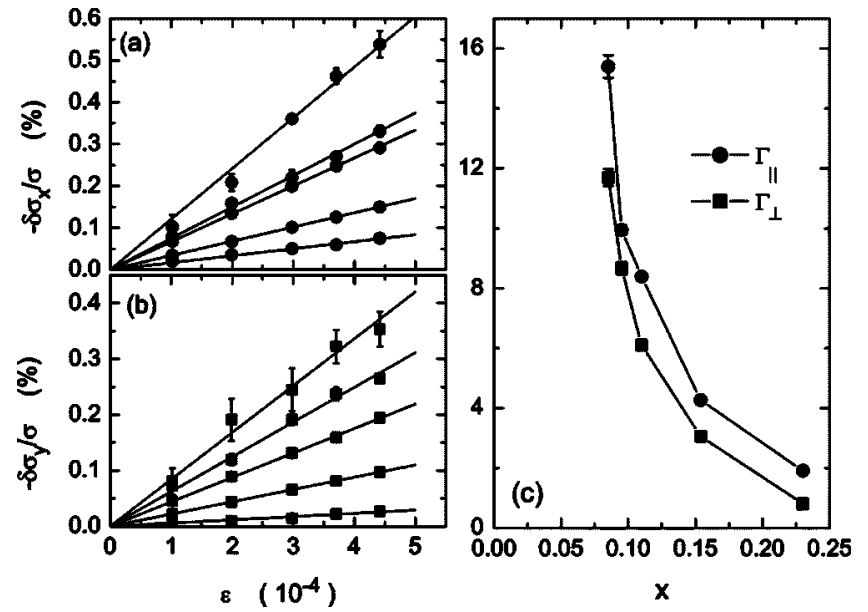

FIG. 10. (a) Relative variation of conductivity along the $x$ axis as a function of applied strain $\varepsilon$ in cantilever bar measurements of the $A 2$ series for different contents $x$ of $\mathrm{RuO}_{2}$. From bottom to top: $x=0.23,0.154,0.11,0.095$, and 0.085 . Solid lines are linear fits to the data. (b) The same of (a) for the case in which the conductivity change is measured along the $y$ axis. (c) Longitudinal $\Gamma_{\|}$, and transverse $\Gamma_{\perp}$, piezoresistive factors obtained by applying Eqs. (32) and (33) to the data of (a) and (b), respectively.

$$
\frac{\delta \sigma_{y}}{\sigma}=-\Gamma_{\perp} \frac{1-2 \nu}{1-\nu} \varepsilon
$$

In Figs. 10(a) and 10(b) we plot the conductivity variations along the $x$ and $y$ direction, respectively, as a function of $\varepsilon$ for the $A 2$ series. The $\mathrm{RuO}_{2}$ volume fractions are $x=0.23$, $0.154,0.11,0.095$, and 0.085 from bottom to top. In the whole range of applied strains, the signal changes linearly with $\varepsilon$, permitting us to extract from the slopes of the linear fits of $\delta \sigma_{i} / \sigma$ vs $\varepsilon$ the values of the longitudinal and transverse piezoresistive factors through Eqs. (32) and (33). The so obtained $\Gamma_{\|}$and $\Gamma_{\perp}$ values of the $A 2$ series are plotted in Fig. 10(c) as a function of $\mathrm{RuO}_{2}$ volume concentration $x$. The main feature displayed in Fig. 10(c) is that the longitudinal and the transverse piezoresistive factors are not much different and both seem to diverge as $x$ approaches the percolation threshold $x_{c} \simeq 0.067$. The fact that $\Gamma_{\|} \sim \Gamma_{\perp}$ is consistent with the vanishing of the piezoresistive anisotropy ratio $\chi=\left(\Gamma_{\|}\right.$ $\left.-\Gamma_{\perp}\right) / \Gamma_{\|}$at the percolation threshold, as discussed in the previous section.

Note instead that the transport exponent for the $A 2$ series is $t \simeq 3.84$ (see Table $\mathrm{I}$ ), that is much larger than $t_{0}=2$. Hence the divergence of $\Gamma_{\|}$and $\Gamma_{\perp}$ as $x \rightarrow x_{c}$ could well be the signature of a tunneling-percolation mechanism of nonuniversality. In order to investigate this possibility we plot in Fig. 11(a) the isotropic piezoresistive factor $\Gamma=\Gamma_{\|}+2 \Gamma_{\perp}$ for the $A 1, A 2, B 1$, and $B 2$ series as a function of $x$. With the exception of the $A 1$ series which displays an almost constant piezoresistive response, the other series clearly diverge at the same critical concentration $x_{c}$ at which $\sigma$ goes to zero. According to the tunneling-percolation theory, $\Gamma$ should follow Eq. (19) which predicts a logarithmic divergence at the percolation threshold when the exponent $t$ is nonuniversal. By using the equivalence between Eqs. (17) and (18) our data 

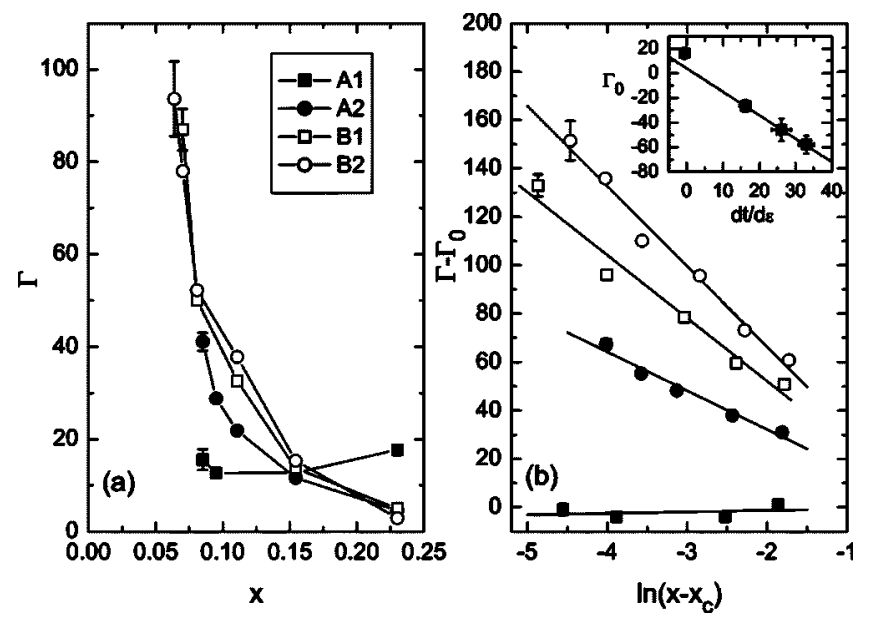

FIG. 11. (a) Piezoresistive factor $\Gamma=\Gamma_{\|}+2 \Gamma_{\perp}$ plotted as a function of $\mathrm{RuO}_{2}$ volume concentration $x$. (b) $\Gamma$ as a function of $\ln (x$ $-x_{c}$ ) and fits (solid lines) to Eq. (34). The fit parameters $d t / d \varepsilon$ and $\Gamma_{0}$ are reported in Table $I$ and in the inset, together with $\Gamma=$ $-1.9 d t / d \varepsilon$ (solid line).

could then be used to verify this hypothesis. This is done in Fig. 11(b) where $\Gamma$ is plotted as a function of $\ln \left(x-x_{c}\right)$ with $x_{c}$ values extracted from the conductivity data. In the entire range of concentrations, and for all the series $A 1, \ldots, B 2, \Gamma$ is rather well fitted by the expression

$$
\Gamma= \begin{cases}\Gamma_{0}, & t=t_{0}, \\ \Gamma_{0}-\frac{d t}{d \varepsilon} \ln \left(x-x_{c}\right), & t>t_{0}\end{cases}
$$

which is Eq. (19) rewritten in terms of $\mathrm{RuO}_{2}$ volume concentration $x$. The $A 1$ series, which has dc exponent $t$ $=2.15 \pm 0.06$ close to the universal value, has no $x$ dependence of $\Gamma$, while the other series $A 2, B 1$, and $B 2$, characterized by nonuniversal exponents, display a logarithmic divergence of $\Gamma$ as $x \rightarrow x_{c}$. This is in agreement with the expectations of the tunneling-percolation theory. Furthermore, as shown in Table I and in the inset of Fig. 11(b), the factor $\Gamma_{0}$ is positive for the $A 1$ series and negative for $A 2$, $B 1$, and $B 2$, in agreement with the results of the last section. For the latter series, $\Gamma_{0}$ behaves as $\Gamma_{0}=-(1.9 \pm 0.5) d t / d \varepsilon$ [see inset of Fig. 11(b)] in accord with the asymptotic relations obtained by EMA, Eq. (26), and by our numerical calculations (31).

The logarithmic divergence of $\Gamma$ for the series having nonuniversal values of $t$ and the corresponding negative values of $\Gamma_{0}$ are features which can be coherently explained by the tunneling-percolation model of nonuniversality. However, in previous studies, this possibility was neglected, and the divergence of $\Gamma$, already reported in Ref. 37 for TFRS and in Ref. 16 for carbon-black-polymer composites, was attributed to a different mechanism independent of the universality breakdown of $t$. This called into play the possibility of having nonzero derivative of the volume concentration $x$ with respect to the applied strain when the elastic properties of the conducting and insulating phases are different. For the particular case of $\mathrm{RuO}_{2}$-based TFRs, one finds that $d x / d \varepsilon$

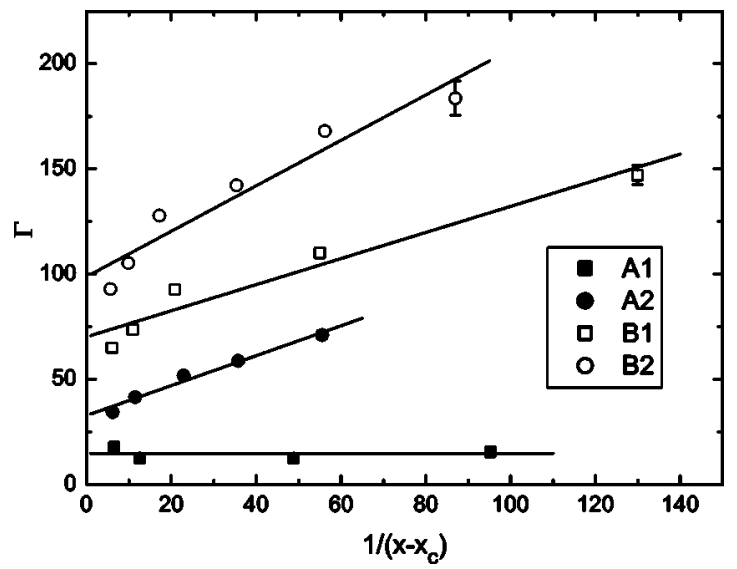

FIG. 12. (a) Piezoresistive factor $\Gamma$ plotted as a function of $1 /\left(x-x_{c}\right)$ (symbols) with tentative fits to Eq. (35) (solid lines). The data related to different series have been shifted vertically by +30 for clarity.

$\simeq-A x$ for small values of the $\mathrm{RuO}_{2}$ concentration $x$. It is easy to show that in this limit $A \propto 1-B_{\text {glass }} / B_{\mathrm{RuO}_{2}}$, where $B_{\text {glass }}$ and $B_{\mathrm{RuO}_{2}}$ are the bulk moduli of the glass and the conducting particles, respectively. Since $B_{\mathrm{RuO}_{2}} \simeq 270 \mathrm{GPa}$ and $B_{\text {glass }} \simeq 40-80 \mathrm{GPa}, A$ is expected to be different from zero and positive. If this reasoning held true, by differentiating Eq. (1) with respect to $\varepsilon$, and by keeping $t$ constant, $\Gamma$ $=-d \ln (\sigma) / d \varepsilon$ would reduce to ${ }^{37}$

$$
\Gamma=\Gamma_{0}+A t \frac{x}{x-x_{c}}=K_{1}+\frac{K_{2}}{x-x_{c}},
$$

where we have defined $K_{1}=\Gamma_{0}+A t$ and $K_{2}=A t x_{c}$. In Fig. 12 we have replotted the $\Gamma$ values of Fig. 11(a) as a function of $1 /\left(x-x_{c}\right)$ with the same values of the critical concentrations $x_{c}$ extracted from the resistivity data. According to Eq. (35), $\Gamma$ should follow a straight line as a function of $1 /\left(x-x_{c}\right)$ which, although being rather correct for the $A 2$ series, is manifestly not true for the $B 1$ and $B 2$ series. In addition, the $A 1$ series remains almost constant, implying that $A=0$ for this case, contrary to the premises of Ref. 37.

In addition to the bad fit with our data, the reasoning of Ref. 37 is based on a misunderstanding of the actual physical meaning of $x$ appearing in Eq. (1). In fact, $x$ should be considered just as an operative estimate of the concentration $p$ of intergrain junctions with finite resistances present in the sample. ${ }^{1,2}$ Current can flow from one end to another of the composite as long as a macroscopic cluster of junctions spans the entire sample. Instead of $x$, an equally valid variable describing the integrain junction probability $p$ would have been the concentration in weight of $\mathrm{RuO}_{2}$, which is manifestly independent of applied strain (and of the elastic properties of the material).

The reasoning of Refs. 16 and 37 is therefore nonconsistent with the physics of percolation. However, one could tentatively argue that the applied strain actually changes the concentration $p$ of junctions by breaking some of the bonds. This situation could be parametrized by allowing a $p$ dependence of $x$ so that $d x / d \varepsilon \simeq(d x / d p)(d p / d \varepsilon)$. Also in this case, 
however, one would end with a $1 /\left(x-x_{c}\right)$ divergence of $\Gamma$ which we have seen to lead to poor fits for our data (Fig. 12). In addition, the values of the applied strains in our measurements are so small $\left(\varepsilon \sim 10^{-4}\right)$ that their effect is that of changing the value of the tunneling junction resistances without affecting their concentration $p$, so that one realistically expects that $d p / d \varepsilon=0$. This is confirmed by the results of Fig. 10(a) which show no deviation from linearity for the entire range of $\varepsilon$ values.

In a previous publication, ${ }^{70}$ we have reanalyzed the piezoresitive data reported in Ref. 37 by assuming a logarithmic divergence of $\Gamma$ rather than the $1 /\left(x-x_{c}\right)$ behavior of Eq. (35). The agreement with Eq. (34) was satisfactory and, furthermore, also in this case we obtained negative values of $\Gamma_{0}$. However, contrary to the present data, all the TFRs used in Ref. 37 were nonuniversal, so it was not possible to establish the disappearance of the logarithmic divergence of $\Gamma$ when $t=t_{0}$.

\section{CONCLUSIONS}

In this paper we have presented conductivity and piezoresistivity measurements in disordered $\mathrm{RuO}_{2}$-glass composites close to the percolation threshold. We have fabricated samples displaying both universal and nonuniversal behavior of transport with conductivity exponents ranging from $t \simeq 2$ for the universal samples up to $t \simeq 3.8$ for the nonuniversal ones. The corresponding piezoresistive responses changed dramatically depending on whether the composites were universal or not. For the composites with $t \simeq 2$, the piezoresistive factor $\Gamma$ showed little or no dependence upon the $\mathrm{RuO}_{2}$ volume fraction $x$, whereas the nonuniversal composites displayed a logarithmic divergence of $\Gamma$ as $x-x_{c} \rightarrow 0$, where $x_{c}$ is the percolation threshold. We have interpreted the piezoresistivity results as being due to a strain dependence of the conductivity exponent when this was nonuniversal. As discussed in Sec. III, a logarithmic divergence of $\Gamma$ is fully consistent with the tunneling-percolation model of nonuniversality proposed by Balberg a few years ago. According to this theory, when the tunneling distance between adjacent conducting grains has sufficiently strong fluctuations, the dc exponent acquires a dependence upon the mean tunneling distance $a$. An applied strain $\varepsilon$ changes $a$ to $a(1+\varepsilon)$ which is reflected in a $\varepsilon$ modulation of the exponent $t$, and eventually to a logarithmic divergence of $\Gamma=-d \ln (\sigma) / d \varepsilon$ at $x_{c}$. By studying an effective medium approximation of the tunneling-percolation model, and by extensive Monte Carlo calculations, we have shown that when $\Gamma$ diverges, the $x$-independent contribution $\Gamma_{0}$ of $\Gamma$ becomes negative, in agreement with what we observed in the experiments.

In view of such agreement between theory and experiments, and given the fact that an alternative explanation based on a strain dependence of the integrain junction concentration is nonphysical and leads to poor fits to our data, we conclude that the origin of nonuniversality in $\mathrm{RuO}_{2}$-glass composites is most probably due to a tunneling-percolation mechanism of nonuniversality. This conclusion is also coherent with the observed correlation between the onset of nonuniversality and the microstructure of our samples, which showed a highly clustered arrangement of the conducting phase when $t=t_{0}$ or a more dispersed configuration when $t$ $>t_{0}$. Although being only qualitative, this picture suggests a possible route for more quantitative analysis on the interplay between criticality and microstructure.

The tunneling-percolation mechanism of universality breakdown could also apply to other materials for which transport is governed by tunneling such as carbon-blackpolymer composites, and experiments on their piezoresistive response could confirm such conjecture. Some earlier data showing diverging piezoresistivity response at the conductorinsulator transition already exist, ${ }^{16,71}$ but their interpretation is not straightforward due to nonlinear conductivity variations as a function of strain or pressure, or even to hysteresis effects.

An interesting issue we have not addressed in the present work is the possible effect of temperature $T$ on the piezoresistivity of disordered composites. As shown in Ref. 54, at fixed concentration of the conducting phase, carbon polyvinylchloride composites display a rather strong enhancement of $\Gamma$ at low $T$. This was interpreted in terms of thermally activated voltage fluctuations across the tunneling barriers. ${ }^{72}$ A qualitatively similar enhancement of $\Gamma$ as the temperature drops is expected also in models based on variable-range hopping mechanism of transport. ${ }^{73}$ It should be pointed out, however, that in these works the problem of connectivity is not included so that the tunneling current flows on a network without percolation characteristics. Nevertheless, the percolation picture (with its corresponding critical exponents) seems to survive well also in the low-temperature tunneling regime. ${ }^{61}$ Hence, a tunneling-percolation theory of piezoresistivity at low temperatures should be formulated by considering percolation networks where the simple tunneling process (9) are combined with additional terms describing, e.g., grain charging effects and/or Coulomb interactions.

\section{ACKNOWLEDGMENTS}

This work was partially supported by TOPNANO 21 (Project NAMESA, No. 5557.2).

\footnotetext{
*Electronic address: claudio.grimaldi@epfl.ch

${ }^{1}$ D. Stauffer and A. Aharony, Introduction to Percolation Theory (Taylor \& Francis, London, 1994).

${ }^{2}$ M. Sahimi, Heterogeneous Materials I: Linear Transport and Optical Properties (Springer, New York, 2003).
}

${ }^{3}$ G. G. Batrouni, A. Hansen, and B. Larson, Phys. Rev. E 53, 2292 (1996).

${ }^{4}$ J.-M. Normand and H. J. Herrmann, Int. J. Mod. Phys. C 6, 813 (1995).

${ }^{5}$ J. P. Clerc, V. A. Podolskiy, and A. K. Sarychev, Eur. Phys. J. B 
15, 507 (2000).

${ }^{6}$ L. Flandin, A. Chang, S. Nazarenko, A. Hiltner, and E. Baer, J. Appl. Polym. Sci. 76, 894 (2000).

${ }^{7}$ A. Dziedzic, Inform. MIDEM 31, 141 (2001).

${ }^{8}$ B. G. Soares, K. M. N. Gamboa, A. J. B. Ferreira, E. Ueti, and S. S. Camargo, J. Appl. Polym. Sci. 69, 825 (1998).

${ }^{9}$ M. B. Heaney, Phys. Rev. B 52, 12477 (1995).

${ }^{10}$ L. Flandin, T. Prasse, R. Schueler, K. Schulte, W. Bauhofer, and J.-Y. Cavaille, Phys. Rev. B 59, 14349 (1999).

${ }^{11}$ Z. Rubin, S. A. Sunshine, M. B. Heaney, I. Bloom, and I. Balberg, Phys. Rev. B 59, 12196 (1999).

${ }^{12}$ S. Nakamura, K. Saito, G. Sawa, and K. Kitagawa, Jpn. J. Appl. Phys. 36, 5163 (1997).

${ }^{13}$ P. Mandal, A. Neumann, A. G. M. Jansen, P. Wyder, and R. Deltour, Phys. Rev. B 55, 452 (1997).

${ }^{14}$ A. Celzard, E. Mc Rae, J. F. Mareche, G. Furdin, M. Dufort, and C. Deleuze, J. Phys. Chem. Solids 57, 715 (1996).

${ }^{15}$ F. Carmona and C. Mourey, J. Mater. Sci. 27, 1322 (1992).

${ }^{16}$ F. Carmona, R. Canet, and S. Delhaes, J. Appl. Phys. 61, 2550 (1987).

${ }^{17}$ H. Czarczynska, A. Dziedzic, B. W. Licznerski, M. Lukaszewicz, and A. Seweryn, Microelectron. J. 24689 (1993).

${ }^{18}$ J. Kubat, R. Kuzel, I. Krivka, S. Bengston, J. Prokes, and O. Stefan, Synth. Met. 54, 187 (1993).

${ }^{19}$ L. J. Adriaanse, H. B. Brom, M. A. J. Michels, and J. C. M. Brokken-Zijp, Phys. Rev. B 55, 9383 (1997).

${ }^{20}$ C. C. Chen and Y. C. Chou, Phys. Rev. Lett. 54, 2529 (1985).

${ }^{21}$ A. Quivy, R. Deltour, A. G. M. Jansen, and P. Wyder, Phys. Rev. B 39, 1026 (1989).

${ }^{22}$ D. van der Putten, J. T. Moonen, H. B. Brom, J. C. M. BrokkenZijp, and M. A. J. Michels, Phys. Rev. Lett. 69, 494 (1992).

${ }^{23}$ R. K. Chakrabarty, K. K. Bardhan, and A. Basu, Phys. Rev. B 44, 6773 (1991).

${ }^{24}$ M. Hindermann-Bishoff and F. Ehrburger-Dolle, Carbon 39, 375 (2001).

${ }^{25}$ S. H. Foulger, J. Appl. Polym. Sci. 72, 1573 (1999).

${ }^{26}$ J. Fournier, G. Boiteux, G. Seytre, and G. Marichy, Synth. Met. 84, 839 (1997).

${ }^{27}$ G. E. Pike, in Electrical Transport and Optical Properties of Inhomogeneous Media, edited by J. C. Garland and D. B. Tanner (American Institute of Physics, New York, 1978), p. 366.

${ }^{28}$ H. C. Angus and P. E. Gainsbury, Electron. Compon. Appl. 9, 84 (1968).

${ }^{29}$ R. W. Vest (unpublished).

${ }^{30}$ W. H. de Jeu, R. W. J. Geuskens, and G. E. Pike, J. Appl. Phys. 52, 4128 (1981)

${ }^{31}$ K. Bobran and A. Kusy, J. Phys.: Condens. Matter 3, 7015 (1991).

${ }^{32}$ A. Szpytma and A. Kusy, Thin Solid Films 121, 263 (1984).

${ }^{33}$ A. Kusy, Physica B 240, 226 (1997).

${ }^{34}$ E. Listkiewicz and A. Kusy, Thin Solid Films 130, 1 (1985).

${ }^{35}$ A. Kubový, J. Phys. D 19, 2171 (1986).

${ }^{36}$ P. F. Carcia, A. Ferretti, and A. Suna, J. Appl. Phys. 53, 5282 (1982).

${ }^{37}$ P. F. Carcia, A. Suna, and W. D. Childers, J. Appl. Phys. 54, 6002 (1983).

${ }^{38}$ A. Dziedzic, Mater. Sci. 13, 199 (1987).

${ }^{39}$ C. Chiteme and D. S. McLachlan, Phys. Rev. B 67, 024206
(2003).

${ }^{40}$ S.-I. Lee, Y. Song, T. W. Noh, X.-D. Chen, and J. R. Gaines, Phys. Rev. B 34, 6719 (1986).

${ }^{41}$ I. J. Youngs, J. Phys. D 35, 3127 (2002).

${ }^{42}$ B. Abeles, H. L. Pinch, and J. I. Gittleman, Phys. Rev. Lett. 35, 247 (1975).

${ }^{43}$ D. Deptuck, J. P. Harrison, and P. Zawadzki, Phys. Rev. Lett. 54, 913 (1985).

${ }^{44}$ Y. P. Mamunya, V. V. Davydenko, P. Pissis, and E. V. Lebedev, Eur. Polym. J. 38, 1887 (2002).

${ }^{45}$ Y. Song, T. W. Noh, S.-I. Lee, and J. R. Gaines, Phys. Rev. B 33, 904 (1986).

${ }^{46}$ Y.-J. Chen, X.-Y. Zhang, T.-Y. Cai, and Z.-Y. Li, Chin. Phys. Lett. 20, 721 (2003).

${ }^{47}$ A. Maaroufi, K. Haboubi, A. El Amarti, and F. Carmona, J. Mater. Sci. 39, 265 (2004).

${ }^{48}$ J. Sun, W. W. Gerberich, and L. F. Francis, J. Polym. Sci., Part B: Polym. Phys. 41, 1744 (2003).

${ }^{49}$ P. M. Kogut and J. P. Straley, J. Phys. C 12, 2151 (1979).

${ }^{50}$ B. I. Halperin, S. Feng, and P. N. Sen, Phys. Rev. Lett. 54, 2391 (1985); S. Feng, B. I. Halperin, and P. N. Sen, Phys. Rev. B 35, 197 (1987).

${ }^{51}$ I. Balberg, Phys. Rev. Lett. 59, 1305 (1987).

${ }^{52}$ I. Balberg, Phys. Rev. B 57, 13351 (1998).

${ }^{53}$ A. Kusy and A. Kolek, Physica A 157, 130 (1989).

${ }^{54}$ E. K. Sichel, P. Sheng, J. I. Gittleman, and S. Bozowski, Phys. Rev. B 24, 6131 (1981).

${ }^{55}$ Y.-M. Chiang, L. A. Silverman, R. H. French, and R. M. Cannon, J. Am. Ceram. Soc. 77, 1143 (1994).

${ }^{56}$ G. E. Pike and C. H. Seager, J. Appl. Phys. 48, 5152 (1977).

${ }^{57}$ C. Canali, D. Malavasi, B. Morten, M. Prudenziati, and A. Taroni, J. Appl. Phys. 51, 3282 (1980).

${ }^{58}$ J. Machta, R. A. Guyer, and S. M. Moore, Phys. Rev. B 33, 4818 (1986).

${ }^{59}$ O. Stenull and H.-K. Janssen, Phys. Rev. E 64, 056105 (2001).

${ }^{60}$ M. Alava and C. F. Moukarzel, Phys. Rev. E 67, 056106 (2003).

${ }^{61}$ I. Balberg, D. Azulay, D. Toker, and O. Millo, Int. J. Mod. Phys. B 18, 2091 (2004).

${ }^{62}$ C. Grimaldi, T. Maeder, P. Ryser, and S. Strässler, Appl. Phys. Lett. 83, 189 (2003); Phys. Rev. B 68, 024207 (2003).

${ }^{63}$ S. Torquato, B. Lu, and J. Rubinstein, Phys. Rev. A 41, 2059 (1990)

${ }^{64}$ C. Grimaldi, T. Maeder, P. Ryser, and S. Strässler, Phys. Rev. B 67, 014205 (2003).

${ }^{65}$ B. Derrida and J. Vannimenus, J. Phys. A 15, L557 (1982).

${ }^{66}$ D. B. Gingold and C. J. Lobb, Phys. Rev. B 42, 8220 (1990).

${ }^{67}$ M. Octavio and C. J. Lobb, Phys. Rev. B 43, 8233 (1991).

${ }^{68}$ K. Adachi, S. Iida, and K. Hayashi, J. Mater. Res. 9, 1866 (1994).

${ }^{69}$ R. P. Kusy, J. Appl. Phys. 48, 5301 (1978).

${ }^{70}$ C. Grimaldi, T. Maeder, P. Ryser, and S. Strässler, J. Phys. D 36, 1341 (2003).

${ }^{71}$ H. Isotalo, J. Paloheimo, Y. F. Miura, R. Azumi, M. Matsumoto, and T. Nakamura, Phys. Rev. B 51, 1809 (1995).

${ }^{72}$ P. Sheng, E. K. Sichel, and J. I. Gittleman, Phys. Rev. Lett. 40, 1197 (1978).

${ }^{73}$ C. Grimaldi, P. Ryser, and S. Strässler, J. Appl. Phys. 88, 4164 (2000). 